\title{
Isolation, Identification and comparatative analysis of SDA and DTM for dermatophytes from clinical samples in a tertiary care hospital
}

\author{
Sowmya Nasimuddin, Appalaraju B, Surendran P, Srinivas CR
}

\begin{abstract}
:
Purpose: The purpose of the study is identify and characterizes different species of dermatophytes from 300 clinically defined cases of ring worm infections. Based upon their morphological features studied by microscopic, culture and biochemical techniques and comparison of Sabouraud dextrose agar (SDA) and Dermatophyte test medium (DTM) for the primary isolation of dermatophytes from the clinical samples. Method: About 300 samples were collected under sterile precautions and observed under microscopy for fungal elements. The culture was done in SDA and DTM and dermatophytes characterization were done by slide culture, bromocresol purple agar, urease test and hair perforation test. Results: Out of 300 samples, 129(43\%) were positive for dermatophytes with majority being Trichophyton mentagrophytes(38.75\%) followed by Trichophyton rubrum (27.13\%). Various species were isolated from skin, hair and nail samples. There was no statistically significant difference between the SDA and DTM $(p<0.01)$ in primary isolation of dermatophytes. Conclusion: With the increasing incidence of severe dermatophytic fungal infections has increased significantly in immunocompetant and also in immunocompromised, there arises a need for effective and rapid diagnosis of such infections.
\end{abstract}

Keywords: Dermatophytes, SDA and DTM, BCP

\section{Introduction:}

Majority of the infections of skin, hair and nail were caused by a group of keratinophilic fungi called the dermatophytes. They include Trichophyton, Microsporum and Epidermophyton. The Trichophyton species usually infect skin, hair and nail. The Microsporum species infect skin and hair, while Epidermophyton species infect skin and nail ${ }^{1}$. Diagnosis of dermatophytic infection is mostly done clinically, but often confused with other skin infections due to topical application of steroid ointments and creams, leading to further misdiagnosis and mismanagement ${ }^{2}$. Hence there arises a need for correct, efficient and rapid laboratory diagnosis of dermatophytes. Direct microscopy with $10-20 \%$ potassium hydroxide $(\mathrm{KOH}) \pm 40 \%$ dimethyl sulfoxide (DMSO) although falsely negative in 5 to $15 \%$ of the cases, was a highly efficient screening technique ${ }^{1}$. The most commonly used culture medium was Sabouraud dextrose agar(SDA) containing antibiotics

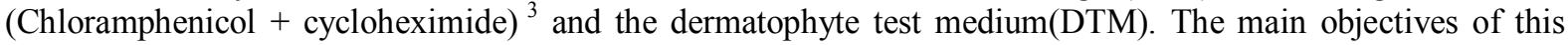
study were identification and characterization of different species of dermatophytes from 300 clinically defined cases of ring worm infections based upon their morphological features studied by microscopic, culture and biochemical techniques and comparison of SDA and DTM for the primary isolation of dermatophytes from the clinical samples.

\section{Materials And Methods:}

Human ethical clearance for the study was obtained from the Institutional ethical committee prior to collection of samples. After informed patient's consent, a total of 300 specimens were collected from the clinically suspected cases of dermatophytic infections from the patients attending the inpatient and outpatient department for one year period. Samples were collected and transported in a sterile black paper, to avoid exposure to moisture and prevent the growth of contaminants. The samples collected were screened for the presence of fungal elements by $20 \%$ potassium hydroxide $(\mathrm{KOH})$ with $40 \%$ dimethyl Sulfoxide Mount $(\mathrm{DMSO})^{4}$. Sabouraud Dextrose Agar (SDA) with antibiotics - Chloramphenicol and Cycloheximide and Dermatophytes Test Medium (DTM) were used for culture of the specimens.

The SDA and DTM were inoculated in duplicate; one incubated at $25^{\circ} \mathrm{C}$ and $37^{\circ} \mathrm{C}$ for 3 weeks. SDA was taken as standard media for primary isolation and compared with DTM (Figure 1a). The growth of dermatophytes in DTM was observed as change in the colour of the medium to red, denoting the shift in $\mathrm{pH}$ to alkaline. Identification of dermatophytes were confirmed by gross morphology of growth, typical microscopic characteristics, supplemented with slide culture, hair perforation test, urease test and growth characters on Bromocresol Purple agar (BCP). The main characters observed in slide culture were - the presence or absence of micro conidia and macro conidia, the nature of their cell walls, their arrangements, the nature of their hyphae. $\mathrm{BCP}$ agar were inoculated and incubated at $30^{\circ} \mathrm{C}$ for 7 days. Growth rates and $\mathrm{pH}$ of the medium were recorded 
at 7 days (Figure 1b). The Christensen's urease agar with 1\% glucose were inoculated and was incubated for 7 14 days at $25^{\circ} \mathrm{C}$. (Figure 2a) Hair from prepubertal age group were collected, sterilized and placed in distilled water with sterile yeast nitrogen base. The colonies of the dermatophytes were introduced just touching the hairs and incubated at room temperature for 14 to 21 days (Figure $2 b$ ).
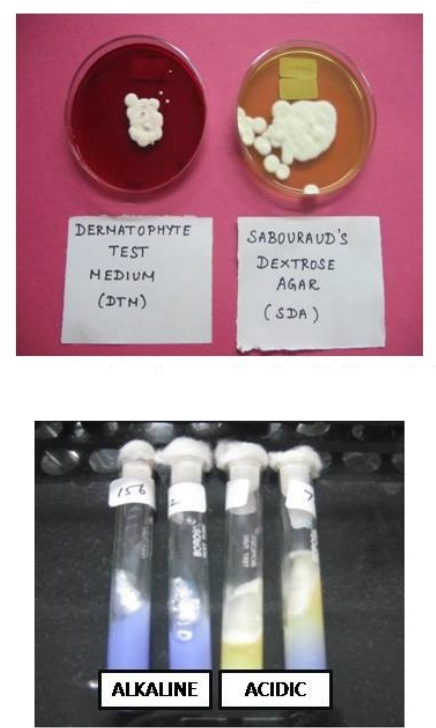

Fig1a - Growth of dermatophytes on SDA and DTM

Fig 1b-Bromocresol purple agar showing acidic and alkaline reaction
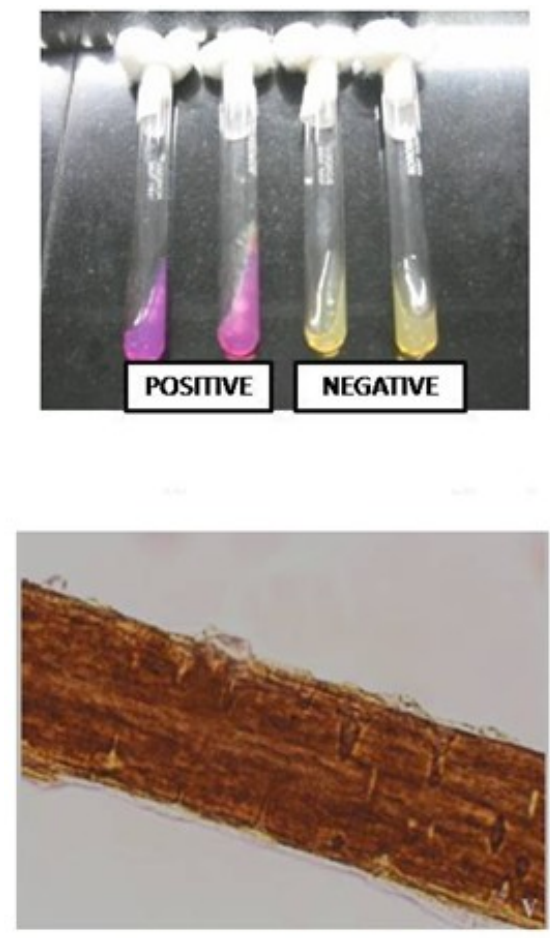

Fig 2a - Urease test - Positive \& negative reactions Fig $2 b$ - Hair perforation test

\section{Results}

The data's were analyzed with Microsoft excel database. Out of 300 samples collected, $103(34.33 \%)$ samples were microscopically positive and $147(49 \%)$ were positive by culture. This includes $129(43 \%)$ dermatophytes and 18(6\%) non- dermatophytic fungi (Table 1). Of the 241(80.33\%) skin samples collected, 90 
$(30 \%)$ were positive for dermatophytes. Thirty three $(11 \%)$ of the $45(15 \%)$ nail samples were positive. Six $(2 \%)$ out of the $15(6 \%)$ hair samples were positive. Majority of the patients had tinea corporis $(24.44 \%)$ followed by tinea cruris(13.33\%). Comparison of SDA and DTM for the primary isolation of dermatophytes from clinical samples was done. Of the $129(43 \%)$ culture positive, $126(42 \%)$ were positive in DTM and $122(40.6 \%)$ were positive in SDA (Table 2). The isolates from the culture medium were subjected to slide culture, hair perforation test, urease test, bromocresol purple agar for identification up to species level. The results of various tests performed for the identification of dermatophytes were shown in table 3

The majority of the isolates were Trichophyton mentagrophytes $(38.75 \%)$ followed by Trichophyton rubrum(11.6\%). Different species of dermatophytes isolated in our study were shown in (Figure 3). The most common causative agents of tinea corporis were T.mentagrophytes $(53.8 \%)$ followed by T.rubrum $(20.5 \%)$ (Figure4). Majority of tinea cruris were caused by T.rubrum (31.8\%). Ectothrix infections were observed with Microsporum gypseum (50\%), T.mentagrophytes (30\%) and T.rubrum (20\%). T.mentagrophytes were found to be the most predominant agent in causing tinea manum, tinea unguinum and tinea pedis (Table 4).

Table 1. Comparison of results of microscopy and culture of positive isolates among dermatophytes infection

\begin{tabular}{|c|c|c|c|}
\hline \multicolumn{2}{|c|}{ Total number of cultures positive } & $147(49 \%)$ \\
\hline \multicolumn{2}{|c|}{ Total number of cultures positive for non dermatophytic fungi } & $129(43 \%)$ \\
\hline & Microscopy & $18(6 \%)$ \\
\hline Culture + & + & $32(10.6 \%)$ & $129(43 \%)$ \\
\hline Culture - & $97(32.3 \%)$ & $165(55 \%)$ & $171(57 \%)$ \\
\hline Total Number & $6(2 \%)$ & $197(65.6 \%)$ & 300 \\
\hline
\end{tabular}

Table 2. Comparison of Sabouraud dextrose agar and Dermatophyte test medium in the primary isolation

\begin{tabular}{|c|c|c|c|}
\hline \multicolumn{3}{|c|}{ Total number of cultures positive for dermatophytes } & $129(43 \%)$ \\
\hline \multicolumn{3}{|c|}{ Culture positive by DTM } & $126(42 \%)$ \\
\hline \multicolumn{3}{|c|}{ Culture positive by SDA } & $122(40.6 \%)$ \\
\hline $\begin{array}{l}\text { SDA } \\
\end{array}$ & DTM & Number & \\
\hline+ & + & 122 & \\
\hline- & + & 4 & \\
\hline+ & - & 0 & \\
\hline
\end{tabular}


Isolation, Identification and comparatative analysis of SDA and DTM for dermatophytes from clinical

Table3. The results of various biochemical tests performed for the identification of dermatophytes

\begin{tabular}{|c|c|c|c|}
\hline & Hair perforation test & Urease test & Bromocresol purple agar \\
\hline $\begin{array}{c}\text { Trichophyton } \\
\text { mentagrophytes }\end{array}$ & Positive & Positive & Alkaline \\
\hline $\begin{array}{c}\text { Trichophyton } \\
\text { rubrum }\end{array}$ & Negative & Negative & Alkaline \\
\hline $\begin{array}{c}\text { Trichophyton } \\
\text { tonsurans }\end{array}$ & Negative & Positive & Alkaline \\
\hline $\begin{array}{c}\text { Trichophyton } \\
\text { equinum }\end{array}$ & Negative & Positive & Alkaline \\
\hline $\begin{array}{c}\text { Trichophyton } \\
\text { meginii }\end{array}$ & Negative & Positive & Alkaline \\
\hline $\begin{array}{l}\text { Trichophyton } \\
\text { ajelloi }\end{array}$ & Positive & Positive & No change \\
\hline Trichophyton violaceum & Negative & Positive & No change \\
\hline $\begin{array}{c}\text { Trichophyton } \\
\text { kanei }\end{array}$ & Negative & Positive & No change \\
\hline $\begin{array}{c}\text { Microsporum } \\
\text { gypseum }\end{array}$ & Positive & Positive & No Change \\
\hline $\begin{array}{l}\text { Microsporum } \\
\text { ferrugineum }\end{array}$ & Negative & Negative & No change \\
\hline $\begin{array}{l}\text { Epidermophyton } \\
\text { flocossum }\end{array}$ & Negative & Positive & Alkaline \\
\hline
\end{tabular}

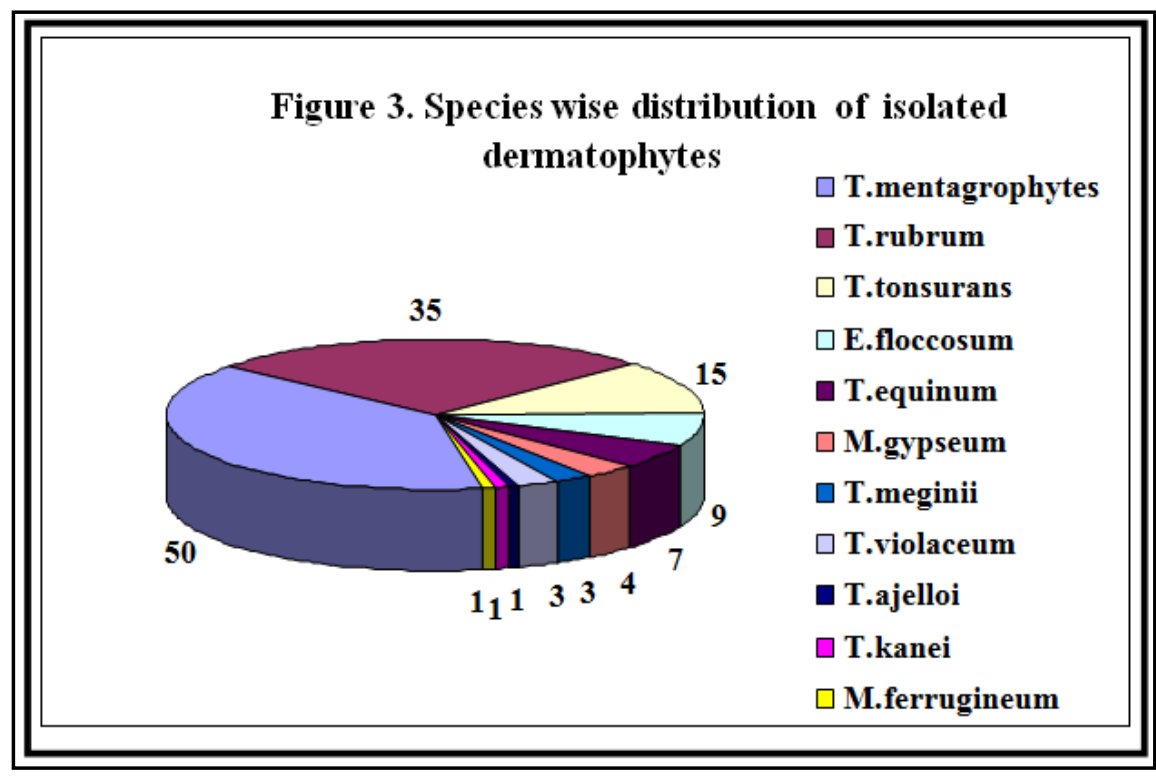

Figure 4. Causative agents of Tinea corporis

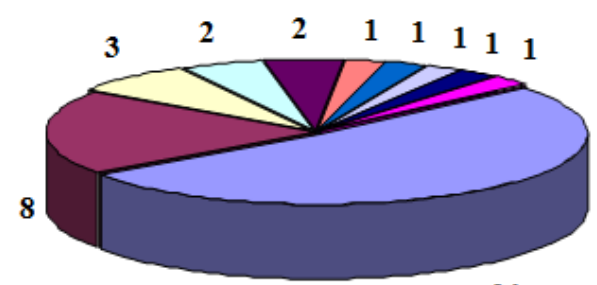

$\square$ T.mentagrophytes

$\square$ T.rubrum

$\square$ T.tonsurans

$\square$ T.violaceum

- E.flocossum

$\square$ T.ajelloi

$\square$ T.kanei

$\square$ M.ferrugineum

M.gypseum

$\square$ T.eeuinum 
Table 4. Showing the causative agents of various dermatophytic infections isolated from our study

\begin{tabular}{|c|c|c|c|c|c|c|c|}
\hline & $\begin{array}{c}\begin{array}{c}\text { Tinea } \\
\text { cruris }\end{array} \\
(19)\end{array}$ & $\begin{array}{l}\begin{array}{l}\text { Tinea } \\
\text { pedis }\end{array} \\
(12)\end{array}$ & $\begin{array}{c}\begin{array}{c}\text { Tinea } \\
\text { manum }\end{array} \\
(7)\end{array}$ & $\begin{array}{c}\begin{array}{c}\text { Tinea } \\
\text { facium }\end{array} \\
(3)\end{array}$ & $\begin{array}{c}\text { Tinea } \\
\text { capitis } \\
(7)\end{array}$ & $\begin{array}{c}\text { Tinea } \\
\text { unguinum } \\
(33)\end{array}$ & $\begin{array}{c}\text { Ectothrix } \\
\text { (7) }\end{array}$ \\
\hline T.mentagrophytes & 3 & 5 & 3 & 2 & 1 & 13 & 2 \\
\hline T.rubrum & 7 & 5 & 1 & - & 2 & 11 & 1 \\
\hline T.tonsurans & 4 & - & 2 & 1 & 2 & 3 & \\
\hline T.meginii & 1 & - & 1 & - & - & 1 & \\
\hline T.equinum & 2 & - & - & - & 1 & 3 & \\
\hline T.violaceum & - & - & - & - & 1 & - & \\
\hline E.floccosum & 2 & 2 & - & - & - & 2 & \\
\hline M.gypseum & - & - & - & - & - & - & 4 \\
\hline & & & & & & & \\
\hline
\end{tabular}

\section{Discussion}

Although dermatophytes do not cause outbreaks or pandemics, the incidence of severe systemic fungal infections had increased significantly, because of the explosive growth in immunocompromised ${ }^{5}$. The present work was conducted to determine the clinical variants of dermatophytes and to compare SDA and DTM for their isolation. The prevalence of dermatophytes among fungal skin infections was found to be $43 \%$. Higher prevalence rates were observed in studies by Kannan et al $(48.5 \%)^{6}$ and Singh et al $(44.61 \%)^{4}$ Lower prevalence rates were also observed in studies by Rajesh et al $32.9 \%{ }^{7}$. Several factors, such as life style, type of the population, migration of people, nature of geographical area and climatic conditions determines their varied distribution. Direct examination by microscopy was highly efficient screening technique as it allows the clinician to start treatment independent of the culture report. The percentage of samples positive by microscopy was $34.3 \%$. Whereas, the rate of positivity by culture was $49 \%$ with $43 \%$ of them being dermatophytes and remaining $6 \%$ were nondermatophytic fungi. The present study shows that culture was more sensitive than microscopy in the diagnosis of dermatophytosis. Our study correlates with other studies by Singh et al where microscopy $(40.76 \%)$ was proved to be less sensitive than culture $(44.61 \%){ }^{4}$

The culture media used for the isolation of dermatophytes were SDA with Chloramphenicol and Cycloheximide and DTM. The rates of positive culture by SDA were $40.6 \%$ and by DTM were $42 \%$. This shows no statistically significant difference between the two medium $(p<0.01)$ for primary isolation from the clinical samples. Our results correlated well with the study by Singh et al, where the two media were proved to be technically good with no statistical difference for the primary isolation ${ }^{4}$. Ringworm has diverse range of clinical manifestation in different areas of the body. The percentage of positive skin, nail and hair samples were $37.3 \%, 73.3 \%$ and $40 \%$ respectively. Many studies have found tinea corporis is the most common among dermatophytic infections ${ }^{6-9}$. Our study also proves that tinea corporis was the most common infection with the high positive rate $(43.33 \%)$ followed by tinea cruris $(24.44 \%)$. The most common isolate from the clinical samples in our study were T.mentagrophytes $(38.75 \%)$ followed by T.rubrum $(27.13 \%)$. But in most other studies the common isolate was T.rubrum followed by T.mentagrophytes ${ }^{4,6-9}$. The reason for more prevalence of T.mentagrophytes in our region could be due to more labor class population with increased migration and climatic conditions.

Various anthrophophilic species like Epidermophyton floccosum, Trichophyton tonsurans and Trichophyton violaceum were reported in our study with $6.9 \%, 11.6 \%$ and $2.3 \%$ respectively. A zoophilic species Trichophyton equinum were reported in $5.4 \%$ of the isolates and Trichophyton ajelloi, a geophilic species from one skin lesion in our study. An anthrophophilic species reported by Summerbell et al, Trichophyton kanei ${ }^{11}$ known to infect keratin tissues. Literature revealed no human reports of this fungus in India, while in our study one case of tinea corporis caused by Trichophyton kanei was noted. Microsporum gypseum were isolated from $3.1 \%$ of our isolates. One case of Microsporum ferrugineum was reported in our study. 
The clinical presentation though typical of ringworm infection is very often confused with other skin disorders particularly due to topical application of steroid ointments and creams, leading to further misdiagnosis and mismanagement ${ }^{2}$. Hence there arises a need for correct, efficient and rapid laboratory diagnosis of dermatophytes. With the proper techniques various species can be identified. And it's the right time to look for the molecular approaches for rapid dermatophytes identification.

\section{References:}

[1]. Rippon JW. 3rd edn. Philadelphia: Saunders; 1988. Medical Mycology: The pathogenic Fungi and the Pathogenic Actinomycetes ; 1988; pp. 140-275.

[2]. Burzykowski, T, Molenberghs G, Abeck D, et al.High prevalence of foot diseases in Europe: results of the Achilles Project. Mycoses. 2003; 46: 496-505.

[3]. Robert R, Pihet M. Conventional Methods for the Diagnosis of Dermatophytosis Mycopathologia 2008; 166:295-306.

[4]. Singh S, Beena PM. Comparative study of different microscopic techniques and culture media for the isolation of dermatophytes. Ind J Med Microbiol. 2003; 21(1):21-24.

[5]. K. J. Kwon-Chung and J. E. Bennett Dermatophytoses. In: Kwon-Chung K J, Bennett J E, editors. Medical mycology, Philadelphia,Pa: Lea \& Febiger; 1992. pp. 105-161.

[6]. Kannan P, Janaki C, Selvi GS. Prevalence of dermatophytes and other fungal agents isolated from clinical samples. Ind J Med Microbiol .2006;24(3) :212-215.

[7]. Rajesh R, Subramainam K, Padmavathy BK, Vasanthi S. Prevalence and species profile of Dermatophytosis among HIV positive patients in ruran referral centre. Indian J Transm disease 2006; 27(2)70 - 74.

[8]. Bindu V, Pavithran K. Clinico - mycological study of dermatophytosis in Calicut. Indian J Dermatol Venereol Leprol 2002; 68:25961.

[9]. Prevalence of zoophilic dermatophytes in the Sarajevo region. Med Arh. 2003; 57(2):101-104.

[10]. Summerbell RC. Trichophyton kanei, sp.nov. a new anthropophilic dermatophyte. Mycotaxon 1987; 28: 509-523. 